\title{
ON SCREENING SAMPLES IN THE LABORATORY \\ AND FACTORS IN FACTORIAL INVESTIGATIONS
}

\author{
Walter T. Federer \\ Cornell University \\ Ithaca, New York
}

BU-926-MA*

May, 1987

*In the Technical Report Series of the Biometrics Unit, Cornell University, Ithaca, New York 14853. 
ON SCREENING SAMPLES IN THE LABORATORY

AND FACTORS IN FACTORIAL INVESTIGATIONS

\author{
Walter T. Federer \\ Cornell University \\ Ithaca, New York
}

\begin{abstract}
Key words: Laboratory samples, group testing, supersaturated fractional replicates, computer simulations, product improvement, sequential responses, search designs.
\end{abstract}

\begin{abstract}
Procedures such as group testing and fractional replication can greatly reduce the workload. The former is especially efficient when the presence of a characteristic in samples is low. Fractional replication can greatly reduce the amount of experimentation when some of the parameters associated with a factorial are nonexistent or negligible. Supersaturated fractional replication can be used to efficiently screen factors when it is suspected that they may not affect a process but it is desired to check this suspicion. There are many types of grouping and pooling material in an investigation. Some of these are discussed as are the interrelationships among the above procedures.
\end{abstract}

\title{
INTRODUCTION
}

There are many types of investigation involved with screening samples in a laboratory for the presence or absence of a characteristic, e.g., disease, contamination, etc. In other areas such as product improvement studies, interest may center on studying factors which may or may not affect the quality of a product. of more recent origin is the widespread use of 
simulation studies using computers. These and other areas are not making use of statistical theory dating back to the $1930 \mathrm{~s}$ and 1940 s. Considerable savings in expense, time, and experimental material can be achieved utilizing these procedures.

Dorfman (1943) proposed a method known as group testing which is described below. A total of $\mathrm{N}$ samples is divided into $G_{1}=N / g$ groups of size $g$. Laboratory analyses to detect presence or absence of a characteristic in a sample are made on the $G_{1}$ groups. For all groups testing positive for the characteristic, individual analyses are made on each sample in the group. Thus, $G_{1}+G_{2} g$ analyses are made, $G_{2}$ is the number of groups testing positive. This number can be considerably less than N, the total number of samples. There are many variations on the basic Dorfman procedure. Two of these are discussed in the next section.

A second procedure that can greatly reduce the number of combinations in a factorial investigation is one called fractional replication. For example, suppose that there are $n$ factors with factor $i$ having $s_{i}$ levels. Then, the total number of combinations is $\mathrm{N}=\Pi_{i=1}^{\mathrm{n}} s_{i}=s_{1} \times s_{2} \times \ldots \times s_{n}$. For $n$ and/or $s_{1}$ large, this may be a very large number. In product improvement studies, each combination may be costly to conduct. Hence, it is imperative to utilize as few combinations as possible to achieve the desired goal. The same is true for computer simulation studies. Here computer time and capacity rather than cost may be the limiting factor. Many statistical simulation studies are beyond the capacity of even a supercomputer. Hence it is important to limit simulations whenever possible. In many situations, saturated main effects fractional replicates of a factorial would suffice to achieve the simulation goals. These ideas are discussed in Section 3. (The reader is referred to Raktoe et al., 1981, for definitions used herein.)

In supersaturated fractional replicates, several factors are grouped together and tested as a group. If the group tests positive (or negative) then one or more of the factors in the 
group is affecting the result of a simulation or product. If the effect of the group of factors is not present, then the entire group may be eliminated from consideration. Some of the ideas related to this type of fractional replication are considered in Section 4.

The last section discusses some of the relationships and economies of the procedures presented. References on the general topic of screening prior to 1962 may be found in Federer (1963). Note that our comments are for screening laboratory samples and that screening per se is not being discussed.

\section{GROUP TESTING FOR SCREENING SAMPLES}

Group testing as proposed by Dorfman (1943) was reportedly proposed for detecting individuals having syphilis in draftees during World War II. It would have been a very difficult task to process each sample individually. Therefore, the $\mathrm{N}$ samples were grouped into $\mathrm{N} / \mathrm{g}=\mathrm{G}_{1}$ groups. A sample of blood was obtained on each member of a group and then a composite sample was made. The analysis for syphilis was made on the composite sample. If the result was negative, none of the members of the group had syphilis. If the result was positive, then an analysis was made on each member of the group. Since the percentage of draftees with syphilis was low, the savings in the number of analyses made was considerable. A result that was previously impractical was now well within the resources available.

Group testing is suitable for all laboratory analyses for which

(i) the presence or absence of a characteristic or a specified amount of a substance is desired,

(ii) the percentage of positives is small,

(iii) the composite sample mean is the same as the mean of the individual samples,

(iv) the compositing does not alter the composition of the samples, and 
(v) the laboratory technique is accurate enough to detect $1 / g$ of the amount of the substance for $g$ the group size used.

When the above conditions are satisfied, group testing can save a considerable amount of time, laboratory equipment, and personnel. Since such savings can be substantial and since laboratory directors and analysts are unaware of the procedure, effort needs to be made to acquaint them with the procedure.

Let us suppose that there are $\mathrm{N}$ samples to be screened for presence or absence of a characteristic. Using a group size of $g$, the $G_{1}=\mathrm{N} / \mathrm{g}$ composite samples would first be processed for the characteristic. Suppose that a percentage $p$ of the $N$ samples are positives and $1-p=q$ are negative. If the presence or absence of the characteristic in one sample is independent of that in another sample, then one may use the binomial expansion of $(p+q)^{g}$ to obtain the proportion of groups expected to be positive, $1 . e, 1-q^{g}$. This means that $G_{1}\left(1-q^{g}\right)=G_{2}$ would be the expected number of groups for which individual analyses would be required. Therefore, the total number of samples to be processed would be

$$
G_{1}+g G_{2}=\frac{N}{g}+N\left(1-q^{g}\right)=N\left(\frac{1}{g}+1-q^{g}\right)
$$

Now when

$$
1 / g=q^{g} \text { or } q=(1 / g)^{1 / g},
$$

It will be a toss-up whether one uses group testing or whether one performs individual analyses on the $N$ samples. This is called the break-even point and is given by equation (2). Note also that

$$
\operatorname{limit}_{g \rightarrow \infty}\left(1 / g+1-q^{g}\right)=1
$$

which says that when $g=N$ one will process $N+1$ samples, $1 . e .$, $\mathrm{N}$ individual analyses and one composite sample of all $\mathrm{N}$ samples. 
The total number of samples to be processed for a group of size $g$ and a percentage of defectives $p$ is

$$
N\left(\frac{1}{g}+1-q^{g}\right)
$$

The value for $g$ which minimizes the total amount of samples processed is obtained as a solution to the following equation

$$
g^{2}=\left(q^{g} \log 1 / q\right)^{-1}
$$

Related results are given by Patel and ottieno (1984a) for grouping factors in a supersaturated fractional designs discussed in Section 4.

Many different group testing plans can be formulated. One of the procedures is described by Sobel and Groll (1959, 1966, 1967). Another is described by Raghavarao and Federer (1973) and Bush et al. (1984). The Sobel-Groll procedure is as follows:

(i) run a test on a composite of $\mathrm{N}$ samples

(i1) if (i) is positive, split into two groups of size $\mathrm{N} / 2$,

(iii) for the positive group(s) in (ii) split into two groups of size $\mathrm{N} / 4$,

(iv) for the positive group(s) in (iii), split into groups of size $\mathrm{N} / 8$,

(v) etc. In that positive groups are split into groups of $\mathrm{N} / 2^{\mathrm{n}}$ at the $\mathrm{nth}$ step.

In order to run $n$ analyses the original sample needs to be relatively large. Note that only so much blood can be taken from one individual, making the Sobel-Groll procedure impractical for this.

The Raghavarao-Federer procedure follows:

(1) run a test on the $\mathrm{N}$ item composite sample; 
(ii) if (i) is positive, partition the $\mathrm{N}$ items into groups of size g (larger than optimal group size); then the $\mathrm{N} / \mathrm{g}=\mathrm{G}$ groups are arrayed in a square or nearly square array of $r$ rows and c columns; then, obtain a composite of $c$ groups in each row and of $r$ groups in each column; then run $r+c$ analyses;

(iii) the intersection-groups from positive rows and columns are the groups containing positive samples; partition each of these groups as in (ii) and repeat (ii) to determine positive samples.

To illustrate, suppose $\mathrm{N}=8,100$. Let $\mathrm{r}=\mathrm{c}=10$ and $\mathrm{g}=81$.

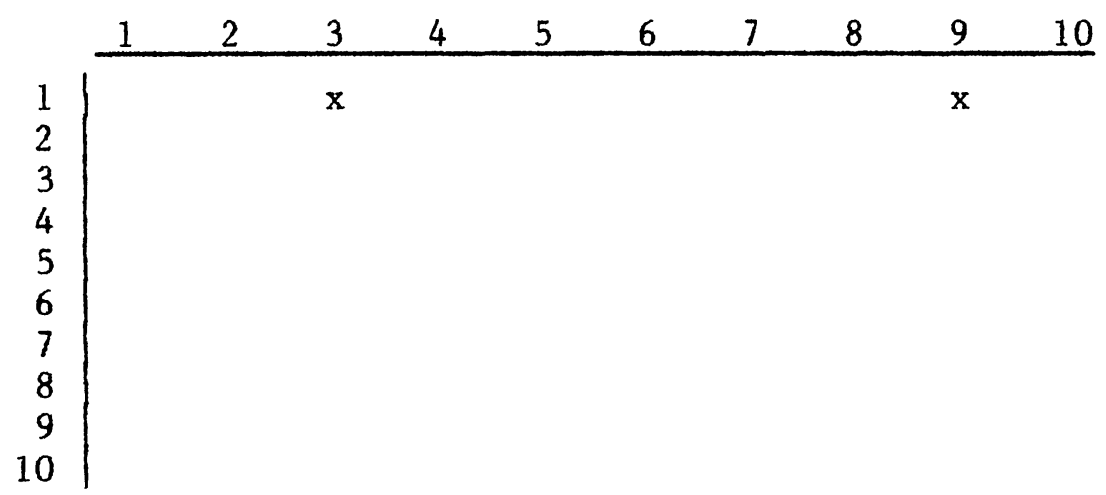

Suppose positive results are obtained for row 1 and columns 3 and 9 , marked by $x$ above. This would indicate that these 2 groups of 81 samples contain positives. Partition these two groups into nine rows and nine columns and test the composites of nine samples in rows and nine samples in columns to possibly determine defective items. The worst configuration for this method is when the positives occur on the diagonal ( $1 . e .$, different rows and columns) of the array.

\section{FRACTIONAL REPLICATION}

Concepts, ideas, construction methods, and properties of fractional replication may be found in Raktoe et al. (1981) and the references contained therein. One of the early references is Finney (1945). Basically the idea of fractional replication of a factorial is as follows. Let $N=\pi_{i=1}^{n} s_{i}$ be the total number 
of combinations of an $n$ factor factorial with the ith factor having $s_{i}$ levels, $B_{N \times 1}$ be the vector of the $N$ parameters associated with the factorial, $\mathbf{Y}_{\mathrm{N} \times 1}$ be the observation vector of combination means associated the factorial, and let $X_{N \times N}$ be the design matrix such that $X \beta=E(Y)$, where $E(\cdot)$ denotes expectation which will be omitted hereafter. Then partition the observation and parameter vectors and the design matrix as follows:

$$
\mathrm{X} \boldsymbol{\beta}=\mathbf{Y}=\left\{\begin{array}{ll}
\mathrm{X}_{11} & \mathrm{X}_{12} \\
\mathrm{X}_{21} & \mathrm{X}_{22}
\end{array}\right\}\left\{\begin{array}{l}
\boldsymbol{B}_{1, \mathrm{p} \times 1} \\
\boldsymbol{B}_{2,(\mathrm{~N}-\mathrm{p}) \times 1}
\end{array}\right\}=\left\{\begin{array}{l}
\mathbf{Y}_{1, \mathrm{p} \times 1} \\
\mathbf{Y}_{2,(\mathrm{~N}-\mathrm{p}) \times 1}
\end{array}\right\},
$$

$0<\mathrm{p} \leq \mathrm{N}$. Then, a fractional replicate may be represented as:

$$
\left(\begin{array}{ll}
X_{11} & X_{12}
\end{array}\right)\left\{\begin{array}{l}
\boldsymbol{\beta}_{1} \\
\boldsymbol{\beta}_{2}
\end{array}\right\}=X_{11} \boldsymbol{B}_{1}+X_{12} \boldsymbol{\beta}_{2}=\mathbf{Y}_{1} \text {. }
$$

Also,

$$
\boldsymbol{B}_{1}+\left(\mathrm{X}_{11}^{\prime} \mathrm{X}_{11}\right)^{-1} \mathrm{X}_{12} \boldsymbol{\beta}_{2}=\left(\mathrm{X}_{11}^{\prime} \mathrm{X}_{11}\right)^{-1} \mathrm{X}_{11}^{\prime} \mathbf{Y}_{1}
$$

when $(\cdot)^{-1}$ exists and a solution for $B_{1}$, given $Y_{1}$ instead of $\mathrm{E}\left(\mathbf{Y}_{1}\right)$, is

$$
\hat{\boldsymbol{\beta}}_{1}=\left(\mathrm{X}_{11}^{\prime} \mathrm{X}_{11}\right)^{-1} \mathrm{X}_{11}^{\prime} \mathbf{Y}_{1}-\left(\mathrm{X}_{11}^{\prime} \mathrm{X}_{11}\right)^{-1} \mathrm{X}_{12} \boldsymbol{\beta}_{2}
$$

If $B_{2}$ is zero or negligible, then one has a solution for the parameter effects in $\beta_{1}$. Otherwise, one estimates the left hand side of (8) and not simply $\boldsymbol{B}_{1}$.

Note that $B_{1}$ may be any set of $p$ parameters and not just those commonly presented in textbooks; the majority of the latter are orthogonal fractions obtained from orthogonal arrays. In many situations a saturated fractional replicate, which is given by (9) when $B_{1}$ and $Y_{1}$ are of the same size with single observations making up $Y_{1}$ and when $\left(X_{11}^{1} X_{11}\right)^{-1}$ exists, (1.e., the number of parameter effects to be estimated is the same as the number of observations), is all that is needed. In other situations, more observations are available than there are para- 
meters to be estimated. The additional observations may be used to obtain an estimate of the error mean square and/or to test for lack of fit. If $\beta_{1}$ consists only of main effects, the fractional replicate is a main effect plan; if $B_{1}$ consists of main effects and all two factor interaction effects, the plan is known as a resolution $V$ fractional replicate; etc. (see Raktoe et al., 1981).

In many experimental situations in product improvement and simulation studies, the observations are obtained sequentially. The combinations for which responses are to be obtained, should be added in such a manner as to have as good a fraction as possible at any stage. Also, the results of each fraction may be studied prior to or during the next run. The one-at-a-time plan (see Anderson and Federer, 1975) may be used to add observations sequentially and to obtain solutions for all parameters up to that point. To fllustrate, a $3^{4}$ factorial set of combinations have been added sequentially in Table 1 . After responses have been obtained for combinations 0000 and 1000 , the mean for 0000 and the $A_{1}$ effect can be estimated; after adding two more combinations 2000 and 0100 and obtaining responses, the additional effects $A_{2}$ and $B_{1}$ may be estimated; etc. for the remainder of Table 1 .

For the first nine observations, the one-at-a-time plan of Table 1 is the one with the largest variance of any design that allows estimation of the mean and main effects. The best plan comes from the following orthogonal array of strength two with the nine columns being the nine combinations:

$\begin{array}{lll}000 & 111 & 222 \\ 012 & 012 & 012 \\ 012 & 120 & 201 \\ 012 & 201 & 120\end{array}$

The effects are estimated after the nine observations for the above combinations have been obtained. If no solution is required until after nine observations have been obtained, there 


\section{TABLE I}

Single degree of freedom parameters in an analysis of variance table together with the observation added sequentially.

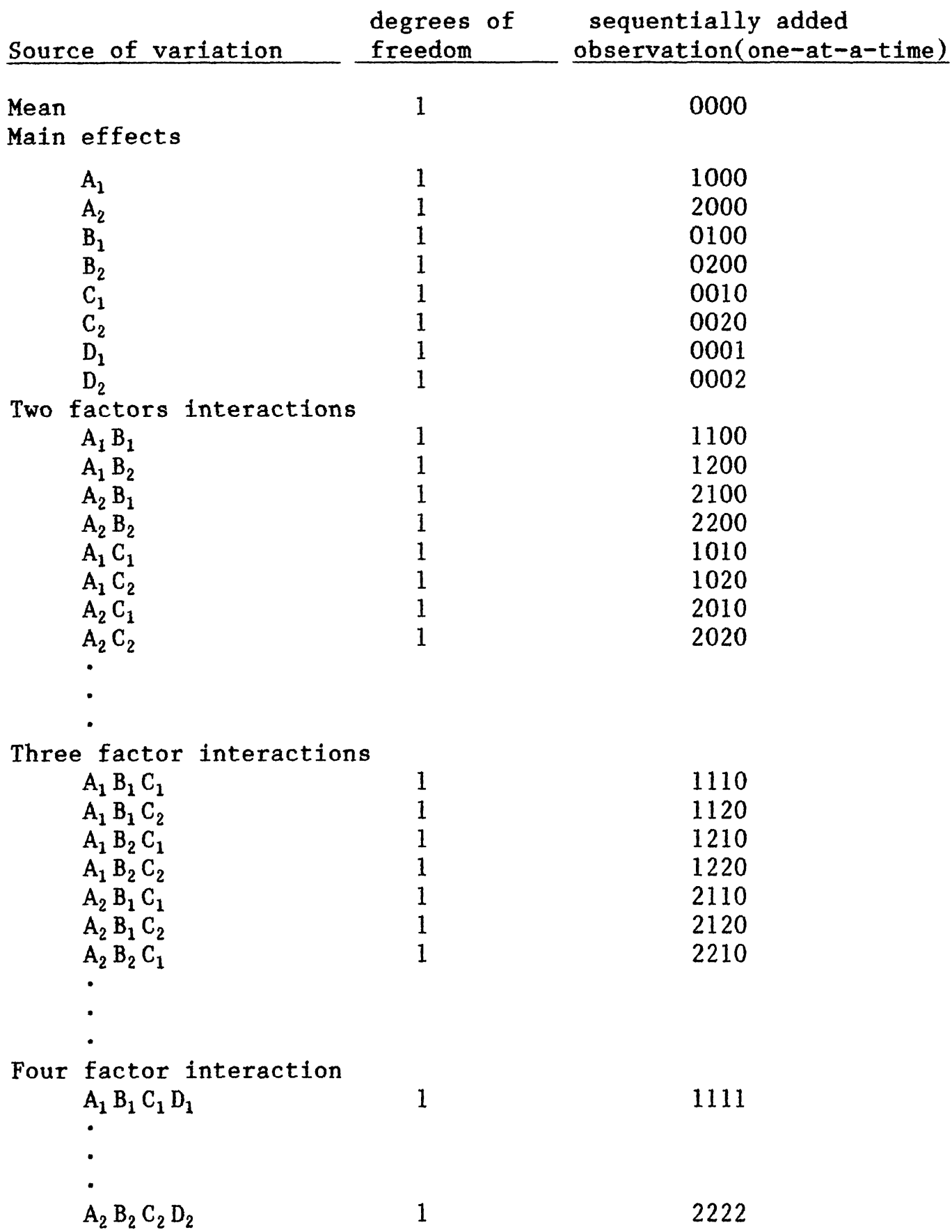


is a considerable difference between the values of the determinants of $X^{\prime} X$ for the two designs, i.e. the ratio of the determinants of $X^{\prime} X$ for the plan in Table 1 to the orthogonal array above, is $3^{-3}$ (see Anderson and Federer, 1985). The ratio of the variances would be the reciprocal of this. Hence, there can be a considerable difference in the variances of estimated effects for the two designs.

The problem of optimal designs in terms of minimal variance when observations have been added sequentially is an unsolved problem for the $\pi_{i=1}^{n} s_{i}$ factorial. Also, the question about alternate designs allowing estimation of all effects up to a given point in an analysis of variance table such as Table 1 , is unresolved.

\section{SUPERSATURATED FRACTIONS FOR SCREENING FACTORS}

Supersaturated fractions of a complete factorial contain fewer observations than there are parameters in $B_{1}$ of equation (9). These designs have been referred to as "group testing" in some of the literature, e.g., Watson (1961), Patel (1962), Li (1962), Kleijnen (1974/75), and Patel and Ottieno (1984a, 1984b). This use for group testing is different from that in Section 2 where the goal was to detect diseased or defective items in a population. Here, the "population" is the set of factors selected by an investigator who categorizes them into groups likely to respond in a similar direction. In the Section 2 group testing the groups are made at random due to lack of knowledge concerning the nature of any of the items in a group. Of course, if by chance there were information available on this, it should be used to decrease the number of groups $G_{1}$ testing positive. In order not to confuse the goals of the investigation, supersaturated fractional designs will not be referred to as grouptesting. One of the earliest papers is by Tukey (1959) and two of the latest papers on grouping factors to form supersaturated 
fractions are by Patel and Ottieno $(1984 \mathrm{a}, 1984 \mathrm{~b})$. The reader is referred to their papers as well as to Kleijnen (1974/75) for detailed results.

To illustrate the above in more detail, suppose that there are sets of parameters in $\beta_{1}$ and $B_{2}$ which are non-zero and which respond in the same direction. A fractional design $\mathbf{Y}_{1}$ would be selected such that in each set, the effects were completely confounded (or aliased) in $\beta_{1}+\left(X_{11}^{\prime} X_{11}\right)^{-1} X_{11}^{\prime} \beta_{2}$. There would be more parameters to estimate than there are observations. The setting in (9) refers to a complete factorial set of effects whereas the usual situation for supersaturated fractional designs would be to have more factors (i.e., main effects) than there are observations. This is illustrated below.

Suppose that the investigator is willing to group factors $E$, $F$, and $G$ with factor $A$, factors $H$ and $I$ with $B$, factor $J$ with $C$, and factors K, L, M, N, and $O$ with D. Suppose that each factor is at three levels. There are four groups of factors, 1.e., $(A, E, F, G),(B, H, I),(C, J)$, and $(D, K, L, M, N, 0)$. The orthogonal main effect plan with nine observations given in the previous section may be used for this. There are $1+15(3-1)=31$ parameters and nine observations. If an effect is detected for the first group, the experimenter only knows that one or more factors $A, E, F$, and $G$ may be producing the effect. He can then run an experiment to detect which of the factors are responsible for the observed effect. Likewise, suppose that no effect was observed for the last group. This could be caused by:

(i) inadequate sample size to detect the response given that it is present,

(ii) effects of some factors are the reverse of others, canceling out the effects,

(iii) interactions are present which act in a direction opposite to main effects, and

(iv) the absence of any effects for these six factors. 
If the first three items above can be ruled out, then the experimenter can conclude that these six factors are not affecting the process and can be eliminated from further consideration.

The steps in constructing a supersaturated fraction of a factorial are:

(i) carefully consider each factor and predict the size of the effect as well as possible,

(ii) plan to use the individual factor without grouping for those factors with large predicted effects,

(iii) group factors together which have responses in the same direction and which may or may not have effects,

(iv) consider which factors or groups are likely to interact,

(v) determine which factors most likely do not affect the responses, and

(vi) for those in (v) use a single level of all these factors in the experiment.

Recently, the author was asked for a treatment design which would accommodate 15 factors each at three levels and seven factors each at two levels. Even a saturated main effect plan with $1+15(3-1)+7(2-1)=38$ runs of the process was inappropriate and impractical. The main effect plan was not considered appropriate since interactions were presumed to be present. It was suggested that the investigator go through the above six steps and return to the statistician after eliminating most of the factors from contention. It was considered likely that several of the factors would fall in group (vi).

Another type of related supersaturated fractional designs and for a different goal than the above has been proposed by Srivastava (1975)(also see Chapter 12 of Raktoe et al. (1981)). These are called search designs. To illustrate, suppose that among a set of $\mathrm{N}$ parameters, an investigator is willing to specify that at most $k<N$ parameters are non-zero. Srivastava 
(1975) and others have given designs for detecting which of the $\mathrm{N}$ effects are non-zero with a minimum number of observations. Search designs are a form of model selection to determine which parameters should be in $B_{1}$ and which should be $\beta_{2}$, the vector of parameters considered to be zero. The reader is referred to the above references and cited references therein for further details on search designs.

Another type of supersaturated fractional factorial is the so-called random balance designs discussed by Satterthwaite (1959). Given that there are $n$ factors to be considered, the levels of each factor are randomly selected and many fewer observations are taken than there are parameters for the $n$ factors. Such designs were proposed for the situation where nothing was known about the individual factors. This situation would not be realistic for most cases in practice where uneven amounts of information on the size of a factor or parameter effect is available.

\section{DISCUSSION}

Our purpose here has not been to give the detailed procedures for group testing and fractional replication but rather to reassert some of the ideas and to indicate their usefulness in investigations. In the course of statistical consulting, the author frequently encounters situations where some type of pooling would greatly reduce the work and/or would make the project feasible. One recent example was a large nutrition dietary - cancer mortality study carried out cooperatively by scientists of Cornell University and the University of Beijeng. The original discussion centered on doing analyses for diet, blood, urine, and possibly hair samples for individual people for about 250 biochemical characteristics. Not enough blood could be extracted from an individual to do the 250 biochemical analyses. Cancer mortality is given by county, sex, and age. The suggestion was made, and followed, that pools of blood samples from 25 individuals of the same sex and commune (there were two communes sampled in each county) be made. This 
suggestion resulted in doing $4 \%$ of the analyses originally proposed. This pooling made the study feasible and provided enough blood for all the biochemical analyses. It should be noted that there are many types of pooling in addition to those discussed in Sections 2 and 4. A pooling procedure for any investigation for which it is considered, should be carefully thought through. There can be pitfalls in pooling samples. These can be avoided by careful planning.

The Ideas in Sections 3 and 4 should be used in computer simulations, as pointed out by Kleijnen (1974/75) and Federer (1987). Also, note that simulations are obtained sequentially in many cases. By summarizing results as they are obtained, it may be possible to stop the simulations sooner than originally planned.

At first thought, group testing and fractional replication may appear to have little in common. However, upon reflection, it should be obvious that one common element is to reduce the total work and make the investigation as efficient as possible. The supersaturated fractional replicates have much in common with group testing. In the former, a search is being made to determine which factors have an effect while in group testing a search is made to find which samples have an effect. As pointed out above there are many situations in which grouping or pooling items (samples, factors, etc.) would greatly reduce the total effort and lead to efficient investigations. One such example has been detailed by Federer et al. (1985). These authors describe a detailed surveillance and control procedure for a disease of dairy cattle. They make use of pooling procedures to greatly reduce the workload in the laboratory, especially in the later stages when the prevalence of the disease is very low.

\section{BIBLIOGRAPHY}

Anderson, D. A. and W. T. Federer (1975). Possible absolute determinant values for square $(0,1)$-matrices useful in fractional replication. Utilitas Mathematica 7, 135-150. 
Anderson, D. A. and W. T. Federer (1985). Zero-one representation and bounds for fractional factorial designs. BU-893-M in the Technical Report Series of the Biometrics Unit, Cornell University.

Bush, K. A., W. T. Federer, H. Pesotan and D. Raghavarao (1984). New combinatorial designs and their application to group testing. Journal of Statistical Planning and Inference $10,335-343$.

Dorfman, B. (1943). The detection of defective members of large populations. Annals of Mathematical Statistics 14, 436-440.

Federer, W. T. (1963). Procedures and designs useful for screening material in selection and allocation; with a bibliography. Biometrics 19, 553-587.

Federer, W. T. (1987). Fractional replication in simulation studies. Communications In Statistics, Simulation and Computation, B16, 233-237.

Federer, W. T., L. C. Clark, E. J. Dubovi, and A. Torres (1985). A surveillance and control procedure for Bovine leucosis and other diseases. BU-877-M in the Technical Report Series of the Biometrics Unit, Cornell University, Ithaca, New York.

Finney, D.J. (1945). The fractional replication of factorial experiments. Annals of Eugenics 12, 291-301. (Correction 15, p. 276).

Kleijnen, J. P. C. (1974/75). Statistical Techniques In Simulation Parts $I$ and $I I$. Marcel Dekker, Inc., New York, pp. 393-450.

Li, C. H. (1962). A sequential method for screening experimental variables. J. American Statistical Association 57, 455-477.

Patel, M. S. (1962). Group-screening with more than two stages. Technometrics 4, 209-217.

Patel, M.S. and J. A. M. Ottieno (1984a). Two-stage groupscreening designs with equal prior probabilities and no errors in decisions. Communications In Statistics - Theor. Meth. 13(9), 1149-1160.

Patel, M.S. and J. A. M. Ottieno (1984b). Optimum two-stage group-screening designs. Communications In Statistics Theor. Meth. 13(21), 2649-2663. 
Rhagavarao, D. and W. T. Federer (1973). Group testing -- A combinatorial approach. BU-473-M in the Technical Report Series of the Biometrics Unit, Cornell University, Ithaca, New York.

Raktoe, B. L. and W. T. Federer (1968). A unified approach for constructing a useful class of non-orthogonal main effect plans in $\mathrm{k}^{\mathrm{n}}$ factorials. Journal of the Royal Statistical Society, Series $B, 30,371-380$.

Raktoe, B. L., A. Hedayat, and W. T. Federer (1981). Factorial Designs. John Wiley and Sons, Inc., New York, xili +209 pages.

Satterthwaite, F. E. (1959). Random balance experimentation. Technometrics 1, 111-137.

Sobel, M. (1967). Optimal group testing. Proc. Colloquium on Information Theory, Bolyai Mathematical Society, Debrecen, Hungary, 411-488.

Sobel, M. and P. A. Groll (1959). Group testing to eliminate efficiently all defectives in a binomial sample. Bell Systems Technical Journal 38, 1179-1252.

Sobel, M. and P. A. Groll (1966). Binomial group testing with an unknown proportion of defectives. Technometrics 8, 631-656.

Srivastava, J. N. (1975). Designs for searching non-negligible effects. In A Survey of Statistical Design and Linear Models (Editor, J. N. Srivastava). North Holland/American Elsevier, Amsterdam, Oxford, and New York, pp. 507-519.

Tukey J. W. (1959). Little pieces of mixed factorials. Biometrics 15, 641-642.

Watson, G. S. (1961). A study of the group screening method. Technometrics 3, 371-388. 